\title{
Recurrent Intracranial Germinoma Refractory to Conventional Irradiation: Effective Chemotherapy Consisting of Cisplatin and Etoposide
}

\author{
-Case Report-
}

\author{
Shuzo OKUNO, Jun-ichiro IsHIKaWA, Kazuhiko NOZAKI \\ and Kazuo YAMAMOTO
}

Department of Neurosurgery, Maizuru Municipal Hospital, Maizuru, Kyoto

\begin{abstract}
Recurrent intracranial germinoma with multiple spinal metastases occurred in a 16-year-old male presenting with persistent headache and visual disturbances. Computed tomography revealed enhanced lesions in the pineal region, anterior horn, and infundibulum. Conventional irradiation achieved remission, but local recurrences requiring further irradiation occurred after 23 months. Magnetic resonance imaging showed multiple spinal metastases 11 months later. Partial removal of the spinal lesions gave a histological diagnosis of typical germinoma. Postoperatively, intracranial recurrences were again detected. Chemotherapy consisting of intravenous cisplatin and etoposide achieved remission and no recurrence has occurred for 12 months.
\end{abstract}

Key words: chemotherapy, cisplatin, etoposide, germinoma, radiation therapy, spinal metastasis

\section{Introduction}

Intracranial germinomas generally respond completely to conventional irradiation with favorable outcomes. Recently, surgical exposure has become acceptable for histological diagnosis and tumor removal even in the pineal region. ${ }^{15,16)}$ Such treatments have achieved 5-year survival rates of $60-80 \%$ in large studies. ${ }^{9,15,19.21)}$ Present research is focused on preventing local and distant recurrence, especially cerebrospinal fluid (CSF) dissemination.

We present a case of intracranial germinoma repeatedly recurring after conventional irradiation, and discuss the problems of maintaining remission and advances in chemotherapy for intracranial germinomas.

\section{Case Report}

A 16-year-old male had a 17-month history of polyuria and polydipsia. In December, 1986, he was admitted with gradually worsening headache, vomiting, and reduced visual acuity. Physical examination revealed a height of $154 \mathrm{~cm}$, a body weight of $35 \mathrm{~kg}$, and hypogonadism manifested with underdeveloped secondary sex characteristics. Neurological examination revealed bilaterally upward gaze pareses, bilateral poor pupillary reaction to light, and bilaterally disturbed visual acuity without field defect.

Serum pituitary hormone levels on admission were normal except for the prolactin level of $97.1 \mathrm{ng} / \mathrm{ml}$ which did not respond to intravenously administered thyrotropin releasing hormone. The growth hormone and adrenocorticotropic hormone responses to intravenous insulin injection were decreased. The antidiuretic hormone level was low at $0.1 \mathrm{pg} / \mathrm{ml}$. Postcontrast computed tomographic (CT) scans demonstrated a well-defined homogeneously enhanced mass in the pineal region containing a calcified fieck. Contrast enhanced masses were also seen in the left anterior horn and infundibulum. The

Received August 27, 1991; Accepted November 20, 1991

Author's present address: S. Okuno, M.D., Department of Neurosurgery, Senboku National Hospital, Sakai, Osaka, Japan. 
ventricles were mildly dilated. A CSF cytological study was negative. The serum levels of alphafetoprotein and human chorionic gonadotropin were normal.

The provisional diagnosis was germinoma. Low doses of local irradiation were delivered. The tumor responded after $16 \mathrm{~Gy}$, and a total of $35 \mathrm{~Gy}$ was delivered, followed by $15 \mathrm{~Gy}$ whole brain irradiation. No spinal irradiation was given. CT scans 2 months later showed that the tumor had completely disappeared and the ventricular size returned to normal. His symptoms resolved, although diabetes insipidus and upward gaze pareses persisted. Desmopressin was given for the diabetes insipidus. Complete remission was maintained for 23 months.

In January, 1989, CT scans demonstrated extensive recurrence in the pineal region, left anterior horn, and suprasellar region (Fig. 1 upper). The tumors disappeared after 20 Gy whole brain irradiation followed by $15 \mathrm{~Gy}$ to the tumor site (Fig. 1 lower). His condition was uneventful except for persistent diabetes insipidus for 11 months.
In January, 1990, he complained of severe lumbago associated with dysesthesia in the bilateral lower extremities and bilateral positive Lasègue's sign. Lumbar myelograms revealed incomplete intradural blocks at the $\mathrm{L} 1 / 2$ and $\mathrm{L} 2 / 3$ levels and a complete block at L5/S1 level (Fig. 2 left). Magnetic resonance (MR) images of the lumbar and sacral regions showed multiple, well-defined homogeneous intensity masses occupying the entire spinal canal at L1, L2, and L5/S1 levels (Fig. 2 right). In January, 1990, a L1-3 laminectomy was performed. Opening the dura exposed a soft grayish mass apparently containing the nerve roots. Most nerve roots were encased and stretched, with a few roots densely adhered to the tumor. So, complete ablation was difficult without sacrificing some. Histological examination of the surgical specimen identified a germinoma of the two-cell pattern (Fig. 3). The tumor cells demonstrated immunoreactivity to placental alkaline phosphatase (PLAP) predominantly on the cell membrane.

A total of $38 \mathrm{~Gy}$ spinal irradiation was given to the
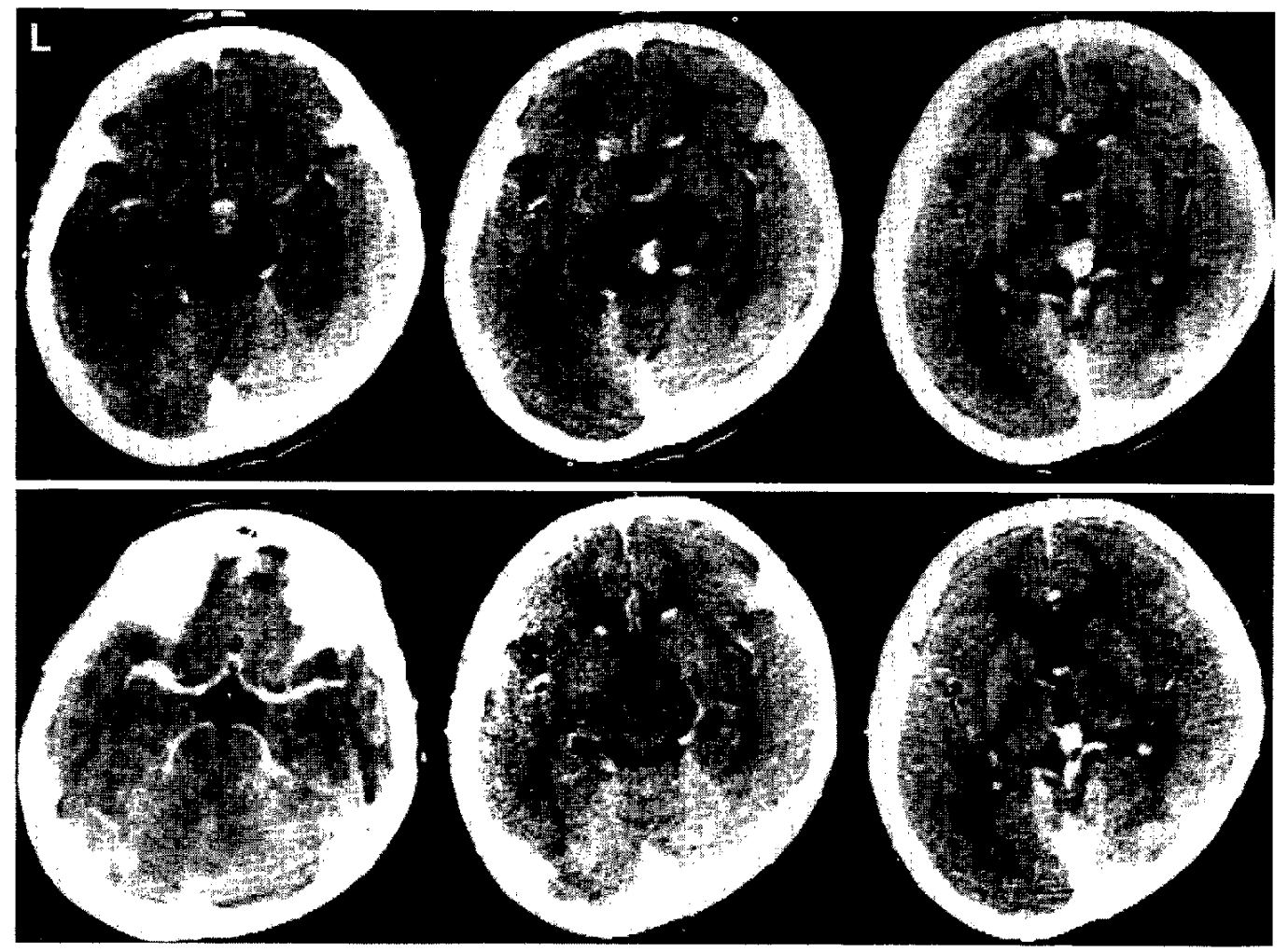

Fig. 1 upper: Postcontrast CT scans, demonstrating multiple high-density masses in the pineal region, left anterior horn, hypothalamus, and suprasellar region. lower: Postcontrast CT scans after $20 \mathrm{~Gy}$ whole brain irradiation and $15 \mathrm{~Gy}$ to the tumor site. The tumors have disappeared with only calcified deposits remaining. 
operative field and sacral tumor site postoperatively. However, MR images showed the sacral tumor to be refractory to irradiation. In March, 1990, a L5-S2 laminectomy and partial removal of the tumor were performed. Intradurally, a yellowish avascular scar and expanded whitish nerve roots were visible. These nerve roots were unlikely to be still functional. We dissected the scar and decompressed the nerve roots
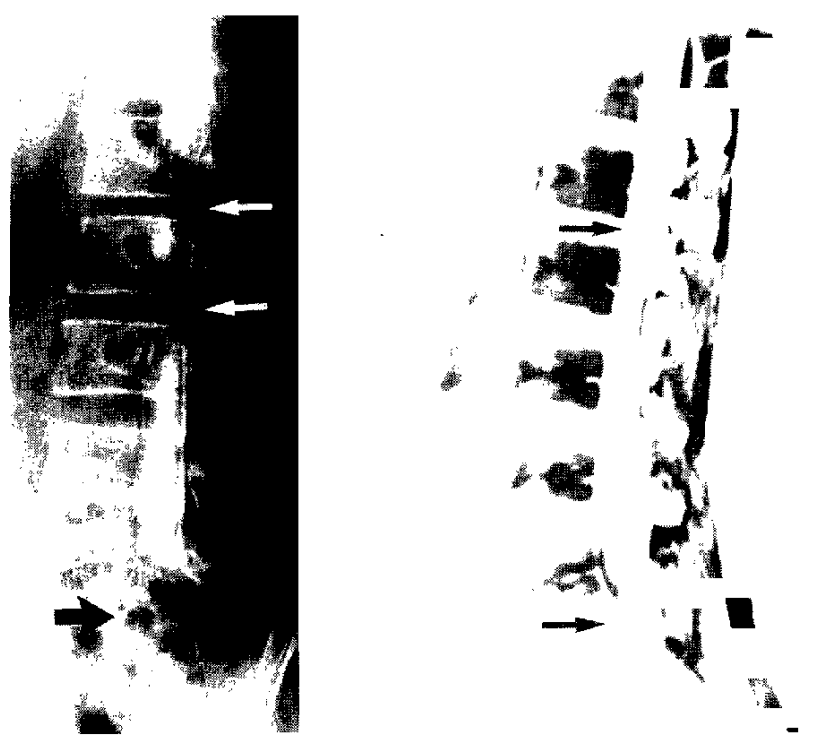

Fig. 2 left: Lumbar myelogram, revealing incomplete intradural blocks at $\mathrm{L} 1 / 2$ and $\mathrm{L} 2 / 3$ levels ( $a r$ rows) and a complete block at L5/S1 level (thick arrow). right: Sagittal $\mathrm{T}_{1}$-weighted MR image of the lumbar and sacral spine with gadolinium enhancement, showing multiple well-defined masses occupying the entire spinal canal at L1, L2, and L5/S1 levels (arrows).



Fig. 3 Photomicrograph of the surgical specimen, demonstrating the two-cell pattern of a typical germinoma. HE stain, $\times 100$.
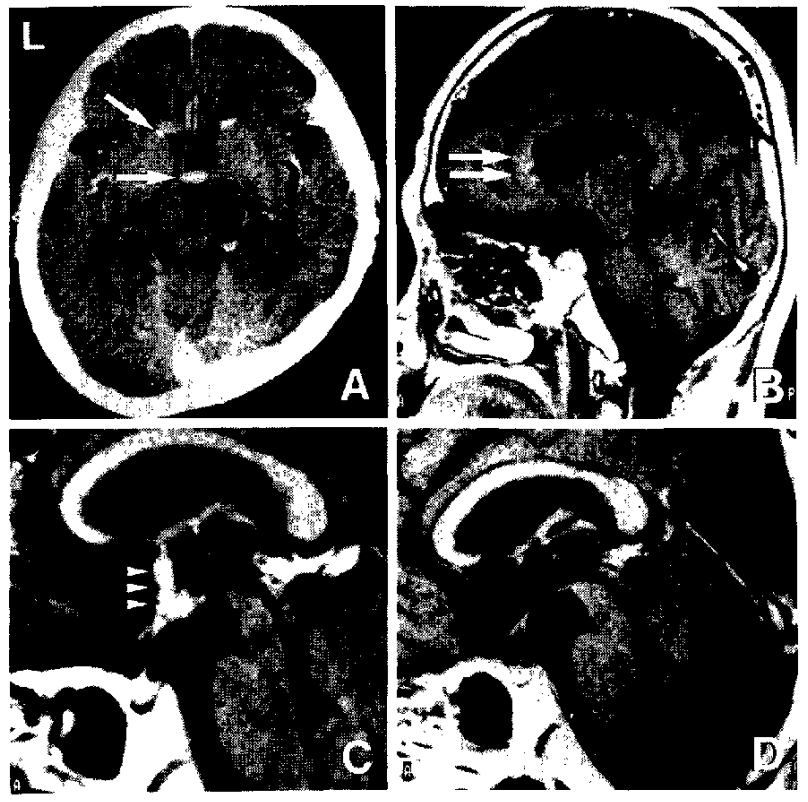

Fig. 4 A: Postcontrast CT scan, showing recurrent tumors in the hypothalamus and left anterior horn (arrows). B: Sagittal $\mathrm{T}_{1}$-weighted MR image with gadolinium enhancement, showing the tumor on the anterior wall of the left anterior horn (arrows). C: Midsagittal $\mathrm{T}_{1}$ weighted MR image, showing the high-intensity tumor in the hypothalamus, extending from the optic chiasm to the anterior wall of the third ventricle (arrowheads). D: Midsagittal $T_{1}$-weighted MR image after chemotherapy, showing no residual tumor.

carefully. Sensory disturbance of the lower extremities was resolved postoperatively, but urinary and fecal incontinence occurred. Follow-up CT scans and MR images demonstrated further metastases in the hypothalamus and left anterior horn (Fig. 4A-C).

In April, 1990, chemotherapy consisting of intravenous $20 \mathrm{mg} / \mathrm{m}^{2}$ cisplatin and $60 \mathrm{mg} / \mathrm{m}^{2}$ etoposide per day was given for 5 successive days. On day 4 , he suddenly complained of nausea and vomiting, and the treatment was discontinued on day 5. Severe renal failure and significant leukopenia appeared but completely resolved after conservative therapy. A second course of chemotherapy, 3 months later, consisted of reduced dosages of cisplatin and etoposide because of his physical condition. The tumors gradually regressed and complete remission has persisted for 12 months (Fig. 4D). 


\section{Discussion}

Intracranial germ cell tumors include several subtypes, but germinomas account for about $65 \%$ of all primary intracranial germ cell tumors. ${ }^{101}$ Germinomas are highly radiosensitive and sometimes cured by radiation therapy alone. Prior to the 1970s, most cases were provisionally diagnosed as germinomas based only on clinical findings, radiological examinations, and CSF cytology.,11) If a dramatic and rapid response to low-dose irradiation was seen, relatively high-dose irradiation was continued up to 50-55 Gy. ${ }^{19)}$ More recent microneurosurgical techniques have decreased the operative morbidity and mortality associated with pineal region procedures, and surgery for histological diagnosis and/or tumor removal ${ }^{(5,16)}$ can achieve favorable 5-year survival rates of $75-80 \% .{ }^{15,19)}$ The recurrence rate is $8-36 \%$ in large studies ${ }^{9,15,19)}$ and the tumor often recurs more than 10 years after remission obtained by irradiation. Germinomas tend to disseminate directly to the hypothalamus and along the walls of the ventricular system, and through the CSF to the spine. Recent research has focused on preventing the local recurrence and metastasis of previously regressed germinomas, including craniospinal irradiation and chemotherapy.

Prophylactic craniospinal irradiation is controversial ${ }^{9,20,22)}$ because of the associated morbidities, particularly in the young, ${ }^{10,18)}$ and because of the low frequency of reported spinal metastasis. ${ }^{19)}$ In our case, the spinal irradiation may have possibly prevented the following spinal metastasis, but not the second intracranial recurrence. Moreover, recurrent tumors appeared to be refractory to irradiation. We suppose that the complete remission in our case could not be achieved only by irradiation.

Chemotherapy, mainly including cisplatin, has significantly lengthened the survival of patients with gonadal $^{6}$ and extragonadal ${ }^{7}$ non-seminomatous germ cell tumors. Intracranial germ cell tumors also respond to such cisplatin-containing regimens. ${ }^{1,12)}$ Neuwelt et al. ${ }^{(4)}$ reported two recurrent germinomas treated with cisplatin, bleomycin, and vinblastine (PVB). Siegal et ll $^{17}$ ) described complete remission of recurrent germinoma with spinal metastasis after PVB treatment. Recently, Allen et al. ${ }^{21}$ suggested that because primary germinomas are highly chemosensitive, radiation doses may be significantly reduced depending on the initial response to chemotherapy. CT showed that 10 of 11 germinomas with a histological diagnosis completely disappeared, and the other patient died not from the tumor but side effects of radiation therapy and chemotherapy. In their series, planned radiation doses could be reduced by $40 \%$ for the tumor and $27 \%$ for the craniospinal region successfully. Recently, Kobayashi et $a l .^{13)}$ found that the combination of cisplatin and etoposide achieved the highest toxicity to Tera 2 and U-251 MG cell lines.

Etoposide, a derivative of semisynthetic podophyllotoxin, is confirmed to be effective against lung carcinoma, ${ }^{3)}$ testicular cancer, ${ }^{41}$ malignant lymphoma, ${ }^{5)}$ melanoma, ${ }^{5)}$ and malignant intracranial germ cell tumors. ${ }^{13}$ This agent inhibits deoxyribonucleic acid synthesis by interrupting cellular mitosis in vitro. ${ }^{8)}$ Myelosuppression secondary to etoposide may be a problem in patients receiving high radiation doses previously, as in the present case. ${ }^{3-5)}$

More studies are required to define the response rate, survival time, and safe clinical use of cisplatin and etoposide for the treatment of germinomas, but chemotherapy may reduce tumor recurrence outside the treated area and enable decreases in radiation doses without compromising the prognosis.

\section{References}

1) Allen JC, Bosl G, Walker R: Chemotherapy trials in recurrent primary intracranial germ cell tumors. $J$ Neurooncol 3: 147-152, 1985

2) Allen JC, Kim JH, Packer RJ: Neoadjuvant chemotherapy for newly diagnosed germ-cell tumors of the central nervous system. $J$ Neurosurg 67: 65-70, 1987

3) Anderson G, Bowyer F, Williams L: Oral VP-16-213 in advanced bronchogenic carcinoma and toxic effects when combined with methotrexate. Thorax 35: 462-464, 1981

4) Bosl GJ, Yagoda A, Whimore WF Jr, Sogani P, Herr H, Vugrin D, Dukeman M, Golbey R: VP-16-213 and cisplatin in the treatment of patients with refractory germ cell tumors. Am J Clin Oncol 7: 327-330, 1984

5) Cecil JW, Quagliana JM, Coltman CA, Al-sarraf M, Thigpen T, Groppe CW Jr: Evaluation of VP-16-213 in malignant lymphoma and melanoma. Cancer Treat Rep 62: 801-803, 1978

6) Einhorn LH, Williams SD: Chemotherapy of disseminated testicular cancer. A random prospective study. Cancer 46: 1339-1344, 1980

7) Hainsworth JD, Einhorn LH, Williams SD, Stewart M, Greco AG: Advanced extragonadal germ-cell tumors. Successful treatment with combination chemotherapy. Ann Intern Med 97: 7-11, 1982

8) Issell BF, Crooke ST: Etoposide (VP-16-213). Cancer Treat Rev 6: 107-124, 1979

9) Jenkin RDT, Simpson WJ, Keen CW: Pineal and suprasellar germinomas. Results of radiation treat- 
ment. J Neurosurg 48: 99-107, 1978

10) Jennings MT, Gelman R, Hochberg F: Intracranial germ-cell tumors: Natural history and pathogenesis. $J$ Neurosurg 63: 155-167, 1985

11) Jooma R, Kendall BE: Diagnosis and management of pineal tumors. $J$ Neurosurg 58; 654-665, 1983

12) Kida Y, Kobayashi T, Yoshida J, Kato K, Kageyama $\mathrm{N}$ : Chemotherapy with cisplatin for AFP-secreting germ-cell tumors of the central nervous system. $J$ Neurosurg 65: 470-475, 1986

13) Kobayashi $T$, Yoshida $J$, Ishiyama J, Noda S, Kito A, Kida Y: Combination chemotherapy with cisplatin and etoposide for malignant intracranial germ-cell tumors: An experimental and clinical study. J Neurosurg 70: 676-681, 1989

14) Neuwelt EA, Frenkel EP, Smith RG: Suprasellar germinomas (ectopic pinealomas): Aspects of immunological characterization and successful chemotherapeutic responses in recurrent disease. Neurosurgery 7: 352-358, 1980

15) Sano K, Matsutani M: Pinealoma (germinoma) treated by direct surgery and postoperative irradiation. A long-term follow-up. Childs Brain 8: 81-97, 1981

16) Shokry A, Janzer RC, Von Hochstetter AR, Yasargil MG, Hedinger $C$ : Primary intracranial germ-cell tumors. A clinicopathological study of 14 cases. $J$ Neurosurg 62: 826-830, 1985
17) Siegal T, Pfeffer MR, Catane R, Sulkes A, Gomori MJ, Fuks Z: Successful chemotherapy of recurrent intracranial germinoma with spinal metastases. Neurology (Cleveland) 33: 631-633, 1983

18) Sklar CA, Grumbach MM, Kaplan SL, Conte FA: Hormonal and metabolic abnormalities associated with central nervous system germinoma in children and adolescents and the effect of therapy: Report of 10 patients. J Clin Endocrinol Metab 52: 9-16, 1981

19) Sung DI, Harisiadis L, Chang $\mathrm{CH}$; Midline pineal tumors and suprasellar germinomas: Highly curable by irradiation. Radiology 128: 745-751, 1978

20) Ueki K, Tanaka R: Treatments and prognoses of pineal tumors: Experience of 110 cases. Neurol Med Chir (Tokyo) 20: 1-26, 1980

21) Waga $S$, Handa $H$, Yamashita J: Intracranial germinomas: Treatment and results. Surg Neurol 11: $167-172,1979$

22) Wara WM, Fellows CF, Sheline GE, Wilson CB, Townsend JJ: Radiation therapy for pineal tumors and suprasellar germinomas. Radiology 124: 221223,1977

Address reprint requests to: S. Okuno, M.D., Department of Neurosurgery, Senboku National Hospital, 2-7-1 Harayamadai, Sakai, Osaka 590-01, Japan. 